\title{
Transkulturelle Kompetenz - Notwendigkeit oder Freifach?
}

\author{
Dieses Fortbildungsangebot bietet Konzepte, um Missverständnisse und Schwierig- \\ keiten bei der Arbeit mit Migrantinnen und Migranten abzubauen. Es will der ärzt- \\ lichen Urkompetenz des Zuhörens und Verstehens zum Erfolg verhelfen.
}

Anke Kayser,

Heinrich Kläui

Dep. Gesundheit und Integration, SRK Bern-Wabern

\author{
Korrespondenz: \\ Anke Kayser \\ Dr. Heinrich Kläui \\ Departement Gesundheit und \\ Integration SRK \\ Werkstrasse 16 \\ CH-3084 Wabern \\ anke.kayser@redcross.ch
}

heinrich.klaeui@redcross.ch
Transkulturelle Kompetenz ist die Fähigkeit, mit Menschen verschiedener Herkunft adäquat umzugehen und ihre individuelle Lebenswelt zu erfassen. Damit wird eine der grundlegenden Fähigkeiten jedes Hausarztes und jeder Hausärztin angesprochen, nämlich der Umgang mit Menschen, die verschiedene soziokulturelle und ökonomische Hintergründe mitbringen. Im Sprechzimmer einer hausärztlichen Praxis tref- diskriminiert. Mögliche Ursachen dafür sind, dass sie unser Gesundheitssystem nicht kennen oder unrealistische Vorstellungen über diagnostische Verfahren und Behandlungsmethoden (Stichwort GanzkörperCT) haben. Aber auch gegenseitige Vorurteile und stereotype Phantasien beeinflussen die Arzt-PatientenInteraktion und erschweren somit die ärztliche Tätigkeit.

\section{Migranten fühlen sich häufig unverstanden, weil sie unser Gesundheitssystem nicht kennen und unrealistische Vorstellungen über Diagnose und Behandlungsmethoden haben}

fen Menschen aufeinander, deren Herkunft, materielle und soziale Situation, Glaube und Überzeugungen ganz unterschiedlich sind. Ärztliche Aufgabe ist es, diesen Menschen in der Beratung und Behandlung gerecht zu werden. Das bedeutet, ihre Vorstellungen, Ängste und Erwartungen zu erfassen und ihnen Lösungen und Behandlungen vorzuschlagen, die für sie nachvollziehbar sind und die ihnen so einleuchtend erscheinen, dass sie die Therapie auch durchführen werden.

So wäre also die mittlerweile oft zitierte transkulturelle Kompetenz ein Neologismus für eine alte hausärztliche Fähigkeit? Warum sollte ein Grundversorger eine Fortbildung zu diesem Thema besuchen?

In den letzten zwanzig Jahren sind Menschen aus vielen, zum Teil weit entfernten Ländern in die Schweiz geflüchtet, um hier Schutz und materielle Sicherheit zu suchen. Diese Menschen sehen wir in unseren hausärztlichen Praxen und stehen ihnen manchmal hilflos gegenüber, sei es, weil wir sie - wörtlich und im übertragenen Sinn - nicht verstehen, sei es, weil unsere Vorstellungen und ihre Realität nicht übereinstimmen.

Hausärztinnen und Hausärzte werden oft mit hohen Erwartungen und Wünschen vonseiten der Migrantinnen und Migranten konfrontiert, die sie nicht erfüllen können oder wollen. Migranten fühlen sich häufig unverstanden, nicht ernst genommen oder

\section{Erklärungsmodelle}

In schwierigen Situationen erweisen sich medizinethnologische Konzepte als hilfreich, um den gedanklichen Raum zu erweitern, sich und das Gegenüber besser zu verstehen und Auswege aus blockierten Situationen zu finden. Arthur Kleinmann prägte den Begriff der Erklärungsmodelle, um die Vorstellungen und Ängste zu beschreiben, die ein kranker Mensch in sich trägt - als Gegenstück zum ärztlichen Erklärungsmodell, das wir in unseren Köpfen tragen [2]. Je mehr und je besser die Erklärungsmodelle von Patient und Arzt zur Sprache kommen und verhandelt werden, desto erfolgreicher die Behandlung - und je mehr die beiden Erklärungsmodelle divergieren, desto grösser wird die Konfrontation und wächst die Unzufrieden-

Als transkulturelle Kompetenz wird die Fähigkeit bezeichnet, individuelle Lebenswelten in der je besonderen Situation zu erfassen, zu verstehen und entsprechende angepasste Handlungsweisen daraus abzuleiten. Transkulturell kompetente Fachpersonen reflektieren eigene lebensweltliche Prägungen und Vorurteile, haben die Fähigkeit, die Perspektiven anderer zu erfassen und zu deuten, und vermeiden Kulturalisierungen und Stereotypisierungen von bestimmten Zielgruppen [1]. 
heit auf beiden Seiten. Ein ähnliches Konzept entwickelte Joseph Levenstein, ein Allgemeinpraktiker aus Südafrika: Durch das Verstehen der Erwartungen, Gefühle und Ängste der Patienten (der Patientenagenda) wird die Sprechstunde zum Ort des Verhandelns zwischen der Arztagenda (Diagnosestellung, «disease») und der Patientenagenda (Krankheitserfahrung, «illness») [3].
Landwirt aus der Region oder einem Mitarbeiter im Management von Novartis?

So genannte kulturelle Unterschiede treten hinter die soziobiographischen Elemente zurück. Zu den sozialen Faktoren gehören die soziale Herkunft des Patienten (städtische oder ländliche Kontexte, Ausbildung und berufliche Tätigkeit), aber auch die soziale Herkunft und Ausbildung/Lebenserfahrung der behan-

\section{Erfahrungsgemäss wird der Einfluss kultureller Faktoren massiv über- schätzt, während soziale Faktoren unterschätzt werden}

\section{Im Fokus: Kultur oder Lebenswelt?}

Handelt es sich um einen Kulturkonflikt? Brauchen wir kulturspezifische Rezepte? Erfahrungsgemäss wird der Einfluss sogenannter kultureller Faktoren häufig massiv überschätzt, während die sozialen Faktoren regelmässig unterschätzt werden. Anders ausgedrückt könnte man auch fragen: Wer steht wem lebensweltlich näher? Ein Arzt, eine Ärztin aus Zürich einer Kollegin aus Damaskus, Syrien? Oder ein Arzt, eine Ärztin aus Basel einem Schweizer, der in der Lonza-Fabrik arbeitet? Ein Arzt, eine Ärztin aus dem Emmental einem

\section{Fortbildungsangebot «Wege aus dem Dilemma»}

Das SRK - gemäss seinen Grundsätzen der Menschlichkeit, Unparteilichkeit und Neutralität - setzt sich für die Gleichheit des Zuganges zum Gesundheitswesen ein, insbesondere der schwachen und verletzlichen Mitglieder unserer Gesellschaft. Um dies zu fördern, hat das Departement Gesundheit und Integration SRK eine Fortbildung für Ärzte und Ärztinnen entwickelt, um Missverständnisse und Schwierigkeiten abzubauen und der ärztlichen Urkompetenz des Zuhörens und Verstehens auch in der Arbeit mit Migrantinnen und Migranten zum Erfolg zu verhelfen. Wenn Sie eine Fortbildungsveranstaltung durchführen oder einen Vortrag zu diesem Thema organisieren möchten, nehmen Sie Kontakt mit uns auf. Unsere Veranstaltungen werden thematisch, inhaltlich und methodisch auf Ihre Bedürfnisse und Fragen abgestimmt, entwickelt und durchgeführt. delnden Fachperson sowie deren politische Ansichten und gegebenenfalls Vorurteile. Diese biographischen Einflüsse prägen darüber hinaus Ausdrucksweisen, Konnotationen beim Sprechen sowie Assoziationen zu bestimmten Begriffen auf beiden Seiten.

Im Konzept der Lebenswelt wird diese Mischung von Einflüssen aller Art (Herkunftsgeschichte, Lebenserfahrungen, Erlerntes und Übernommenes, Erfahrenes und Gelesenes) erfasst, die das Individuum ausmacht, das vor uns sitzt. Anstatt bei Problemen die «Kultur» in den Vordergrund zu rücken, ist es sinnvoller, die unterschiedlichen Lebenswelten zu erfassen.

\section{Literatur}

1 Domenig D (Hrsg.). Transkulturelle Kompetenz. Lehrbuch für Pflege-, Gesundheits- und Sozialberufe. Bern: Hans Huber; 2007.

2 Kleinmann A, Eisenberg L, Good B. Culture, Illness, and Care; Clinical Lessons from Anthropological and Cross-Cultural Research. Ann Int Med. 1978;88:252-8.

3 Levenstein JH, McCracken EC, McWhinney IR, Stewart MA, Brown JB. The patient-centered clinical method. A Model for the doctor-patient interaction in family medicine. Family Practice. 1986;3:24-30. 